\title{
Stability Test Results on the Aluminum Stabilized Superconductor for the Helical Coils of LHD
}

\author{
Nagato Yanagi, Takashi Satow, Toshiyuki Mito, Shinsaku Imagawa, Kazuya Takahata, Akifumi Iwamoto, \\ Hirotaka Chikaraishi, Satarou Yamaguchi, Sadao Satoh, Osamu Motojima, and the LHD Group \\ National Institute for Fusion Science, 322-6 Oroshi-cho, Toki, Gifu 509-5292, Japan \\ Soh Noguchi and Atsushi Ishiyama \\ Waseda University, 3-4-1 Ohkubo, Shinjuku-ku, Tokyo 169-8555, Japan
}

\begin{abstract}
Stability tests have been carried out on short samples of the aluminum/copper stabilized composite-type superconductors developed and used for the pool-cooled helical coils of the Large Helical Device. The waveform of the longitudinal voltage initiated by resistive heaters shows a short-time rise before reaching a final value, which seems to correspond to the diffusion process of transport current into the pure aluminum stabilizer. The propagation velocity has a finite value even for the transport current being lower than the recovery current, and it differs depending on the direction with respect to the transport current.
\end{abstract}

\section{INTRODUCTION}

The Large Helical Device (LHD) is a fusion experimental device that has been completed with all superconducting coil systems [1]. The two helical coils have the major radius of $3.9 \mathrm{~m}$ and the average minor radius of $0.975 \mathrm{~m}$ with a poloidal pole number of 2 and a toroidal pitch number of 10. They have been successfully cooled down with liquid helium at $4.4 \mathrm{~K}$ and excited up to the central toroidal field of $1.5 \mathrm{~T}$ through the first campaign of LHD plasma experiments. The toroidal field will be raised up to $3 \mathrm{~T}$, in the near future, that will correspond to the specified operation point in the Phase I condition of LHD. Superfluid helium of $1.8 \mathrm{~K}$ will be later utilized in the Phase II operations, and the central field will reach up to $4 \mathrm{~T}$ with a maximum stored magnetic energy of $1.6 \mathrm{GJ}$.

A pool-cooled composite superconductor of $\mathrm{NbTi} / \mathrm{Cu}$ compacted strands with pure aluminum/copper stabilizer was selected to be wound up as the helical coils, incorporating the advantages of high cryogenic stability and mechanical flexibility at enough strength. The conductor size, $12.5 \mathrm{~mm} \times 18.0 \mathrm{~mm}$, as well as the inner configuration were carefully chosen, as shown in Fig. 1, through an intensive $R \& D$ program [2]-[4].

One of the main problems we met during the development of this conductor was the finding of unexpectedly low recovery current, which was due to the effective magnetoresistivity of the copper/aluminum composite stabilizer.

Manuscript received September 14, 1998.

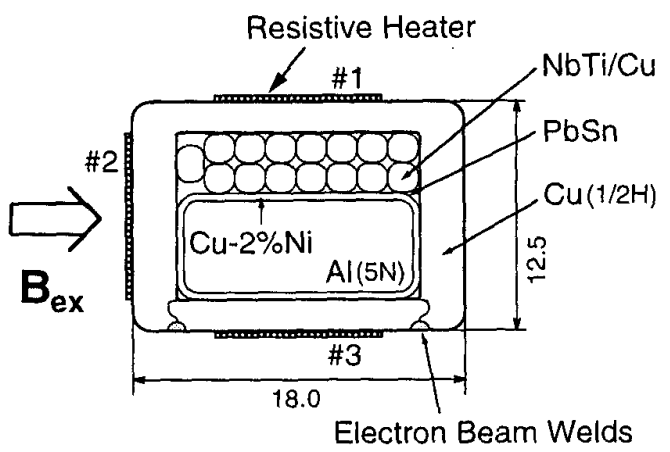

Fig. 1. Cross-sectional view of the superconductor developed and applied to the helical coils of LHD.

This phenomenon could be well explained later by the so called "Hall current" generation model for metal-metal composites with different conductive materials $[5,6]$. In order to reduce the spurious "Hall current" and hence the effective magnetoresistivity, a $\mathrm{Cu}-2 \% \mathrm{Ni}$ alloy clad (the resistivity approximates $2.5 \times 10^{-8} \Omega \cdot \mathrm{m}$ at $7 \mathrm{~T}$ and $4.4 \mathrm{~K}$ ) was installed around the pure (" $5 \mathrm{~N}$ ") aluminum core (Fig. 1). Short sample tests have been carried out to investigate the characteristics of this conductor. The critical current measurements and the stability tests were the most important items.

An important peculiarity of superconductors stabilized with pure aluminum is regarding dynamic stability which is caused by a rather long diffusion time of transport current from superconducting strands into the aluminum because of the high electrical conductivity of the latter. For example, in the experiments for a SMES conductor, there has been observed, as it is called, a traveling normal zone $[7,8]$, which showed that the initiated normal zone propagated forward and recovered at the back end with a typical time scale corresponding to the current diffusion time in the aluminum stabilizer. Appearance of such a traveling normal zone should be treated as an important issue not only from the view point of cryogenic stability, but also of continuous heat load on a magnet system as well. 
In this paper, the stability test results of the LHD helical coil conductors are described, mainly focusing on the transient characteristics of the aluminum stabilizer.

\section{EXPERIMENT}

\section{A. Experimental Setup}

Stability tests have been carried out for a number of short samples of the LHD helical conductors through an $R \& D$ program as well as in the inspection tests for the final products prior to the coil winding process. They were examined in the superconductor test facilities with a $9 \mathrm{~T}$ split coil, $100 \mathrm{kA}$ current leads and $75 \mathrm{kA}$ DC power supplies. A short sample consists of two or four conductors, each about $2 \mathrm{~m}$ long, soldered together in series. The bias field is given by the split coil (of outer diameter $907 \mathrm{~mm}$ ) which has a $90 \%$ flat top field region of about $250 \mathrm{~mm}$ [2], and the short samples were vertically inserted into the bore ( $100 \mathrm{~mm}$ by $550 \mathrm{~mm}$ ) of this split coil. For some samples, the conductors were minutely bent in order to simulate a coil lead section and this lengthened the effective field distribution (for about $50 \%$ ) than that for a straight sample. A number of voltage taps, thermometers and stainless steel resistive heaters were attached onto the conductor surface, all imbedded beneath GFRP spacers which defined the exposure rate of conductors as $50 \%$ in the standard configuration. It is notable that as for the stability test, it was possible to examine each conductor individually by placing resistive heaters at the sample centers which coincided with the geometrical center of the split coil.

\section{B. The Stability Test Results}

In the set of stability tests for the LHD helical conductors, intensive attention was paid mainly to the recovery current. Therefore, the stability tests were usually carried out by supplying the heater energy with low power and long duration (up to 1 second). Fig. 2 shows an obtained example of the required energy for initiating a normal zone as a function of the transport current. As may be inferred from Fig. 2, the initiated normal zone collapsed, and the sample recovered into the superconducting state at a transport current that was lower than $13.7 \mathrm{kA}$ even if a substantial amount of heater energy was supplied. On the other hand, the required energy for initiating a normal zone approaches to zero when the transport current reaches up to the measured critical current of $22 \mathrm{kA}$ under the external transverse field of $7 \mathrm{~T}$.

In Fig. 2, the "recovery current" was determined at which the initiated normal zone was observed to disappear as we gradually decreased the transport current after having a normal zone stagnation. Fig. 3 shows an example of the measured longitudinal resistance of the stagnated normal zone using the potential taps distributed along the conductor sample. The phenomenon of the normal zone

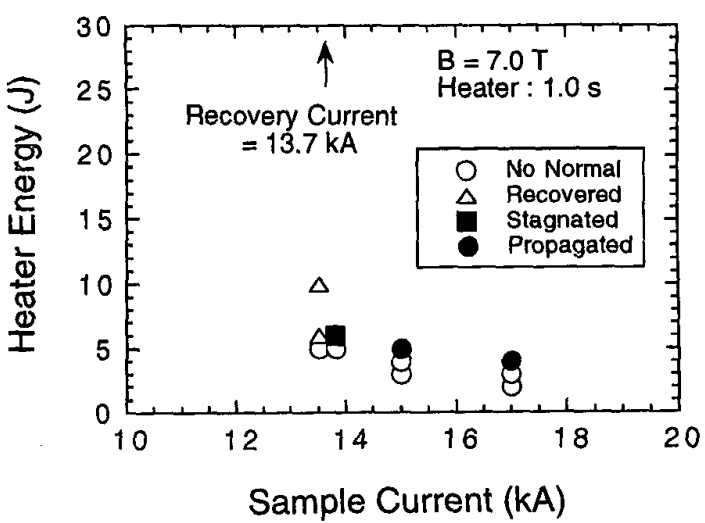

Fig. 2. Dependence of the critical energy of heater on the transport current of the superconductor sample.

stagnation might be brought about by the fact that the bias magnetic field has a finite spatial distribution [9]. The window of transport current for observing the stagnation is typically $2-3 \mathrm{kA}$ depending on the recovery current value and the bias field. The recovery current has been measured with this procedure for more than 40 samples from the view point of quality control, and the results are summarized in [4].

The required heater energy was examined also by varying the direction of heat input as well as the duration of power. As can be seen in Fig. 4., the required heater energy turns out to be almost three times higher when the energy was given from the aluminum side than with a case from the strand side. This observation can be explained by the high longitudinal heat conductivity of the pure aluminum stabilizer, which should significantly reduce the heating power density required to increase the temperature of the superconducting strands to be higher than the critical temperature. The required heater energy decreases when the duration of heater pulse is shortened both for the aluminum side and strand side. For a pulse

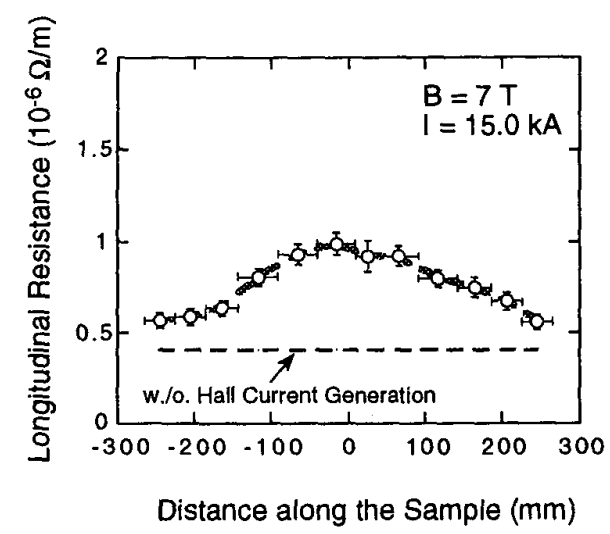

Fig. 3. Distribution of the measured longitudinal resistance along the conductor sample during a normal zone stagnation. 


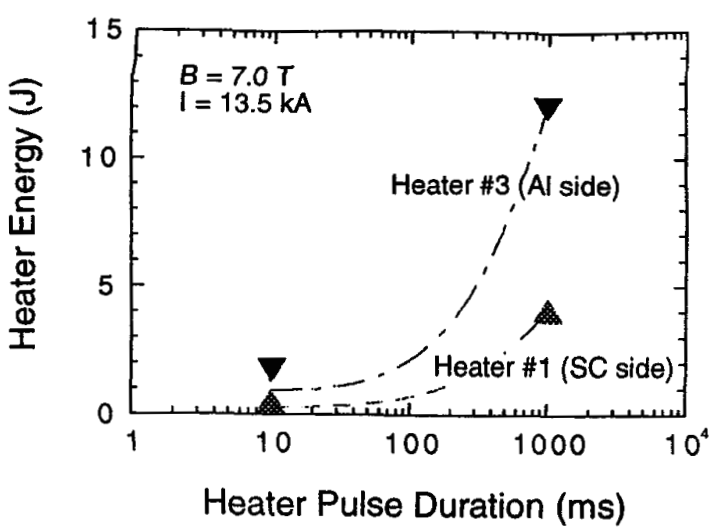

Fig. 4. The critical energy of heater vs heater pulse duration. The dashed and dash-dotted curves indicate the obtained dependence of the required heater energy on the pulse duration through a similar experiment with slightly different conditions .

duration less than $20 \mathrm{~ms}$, this critical energy becomes almost constant, as was observed in a similar experiment for the same type of conductor. The precise value for the minimum quench energy is hard to be determined for the type of resistive heaters used in the present experiments due to their own heat capacity [10].

\section{Transient Behaviour of Normal Zone}

Fig. 5 shows a typical waveform of the longitudinal voltage measured with a pair of potential taps located at the sample center (of distance $47 \mathrm{~mm}$ ). As can be seen in Fig. 5, the voltage shows a peak, nearly $100 \mathrm{~ms}$ wide, and then it decreases to reach a steady-state value (the normal zone stagnation). This peak of the voltage seems to correspond to the magnetic diffusion process in the pure aluminum core with a characteristic time scale $t_{m}$ of

$$
t_{m}=\frac{\mu_{o} d^{2}}{\pi^{2} \rho_{A l}},
$$

which can be estimated based on a model for onedimensional magnetic diffusion process. Here, $d$ is the width of the aluminum core, and $\rho_{A l}$ is the aluminum resistivity. By substituting the parameters of $d=5 \mathrm{~mm}$ and $\rho_{A l}=3.8 \times 10^{-11} \Omega \cdot \mathrm{m}$ (at $4.4 \mathrm{~K}$ and $7 \mathrm{~T}$ ) for our conductor, $t_{m}$-value is estimated at $84 \mathrm{~ms}$, which seems to be fairly close to the time constant value of $97 \mathrm{~ms}$ determined from the longitudinal voltage measurements.

The propagation velocity of normal zone is plotted as a function of transport current as shown in Fig. 6. As may be inferred from Fig, 6 , the propagation velocity depends on the direction of the propagation, i.e., about twice faster in the downstream side of the transport current than that in the upstream side. Here it should be noted that this effect does not depend on the actual spatial direction of the sample, i.e., the direction of gravity, and the reason for this observation has not been clarified so far and leaves under study.

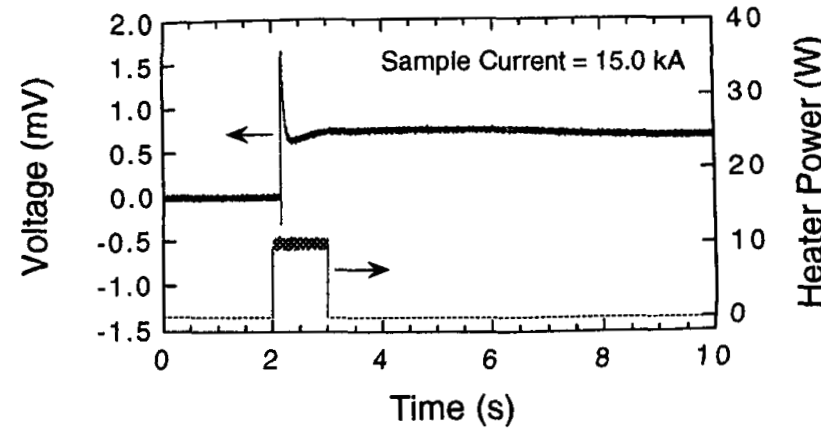

Fig. 5. Typical waveform of the longitudinal voltage in the sample.

Another important point seen in Fig. 6 is the fact that the propagation velocity has a finite value even in some range below the recovery current, which suggests there is a noticeable discrepancy between the minimum propagating current and the recovery current. This can be understood when considering the characteristics of the pure aluminum/copper composite stabilizer that its cross-section cannot be fully used as a current conducting medium during the time period of the order of $100 \mathrm{~ms}$ necessary for the current (electro-magnetic) diffusion process to be completed just after the superconducting-normal transition of the strands. Thus, the heat generation in the vicinity of a normal zone front becomes higher than that in the central region, and such a normal zone should once propagate, though the transport current is lower than the recovery current. Since there have not been enough data below the recovery current, it is hard to determine precisely the minimum propagating current at which the propagation velocity must become zero. As was found in [7], there is a possibility of observing a traveling normal zone also for our conductor in this current range. Probably, this will be examined for longer conductor samples, i.e., representative coil samples, which will be tested in the nearest future.

In order to clarify the experimentally observed characteristics in the stability test, high-accuracy numerical

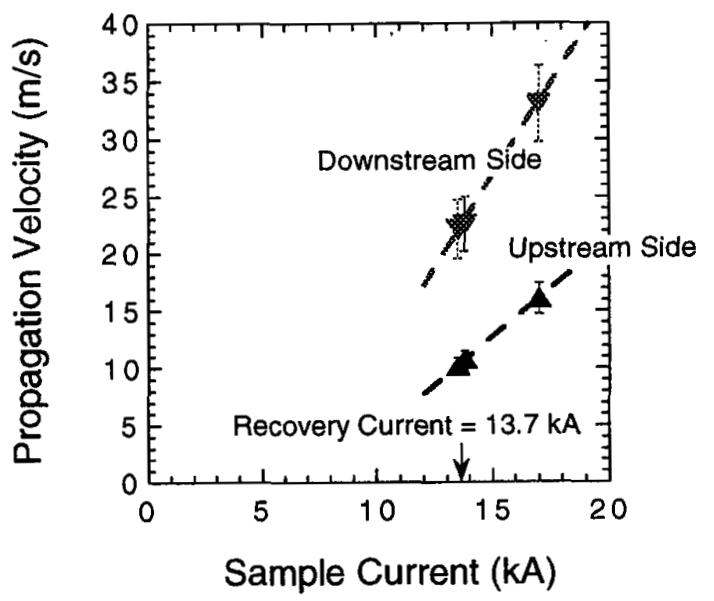

Fig. 6. The propagation velocity dependence on the transport current at bias transverse magnetic field of $7 \mathrm{~T}$ maximum. 
calculations have been conducted, especially focusing on the transient current diffusion process in the stabilizer by adopting an FEM approach [11]. It enabled to explain, at least qualitatively, some of the above-mentioned effects. At the same time, the observed difference of the propagation velocity depending on the direction of transport current has not been treated yet in the framework of this approach [11] leaving under further perfection.

\section{CONCLUSIONS}

The stability characteristics of the aluminum and copper stabilized superconductors developed and applied to the helical coils of LHD have been studied experimentally. Transient behaviour of normal zones initiated by resistive heaters has been investigated for short samples under the specified bias magnetic field. To determine the recovery current, a stagnation point of longitudinal voltage has been found. A short-time drastic rise (peak) of longitudinal voltage has been observed just after the superconductingnormal transition. Presumably, it owes to the relatively slow diffusion process of the transport current into the pure aluminum core. It has been found that the propagation velocity depends on the direction of transport current; the velocity towards the downstream side of transport current at least doubles that towards upstream side.

\section{ACKNOWLEDGMENT}

The authors wish to thank Dr. T. Senba and Dr. A.V. Gavrilin for the fruitful discussions regarding the normal zone propagation. The authors greatly appreciate the efforts of HITACHI Ltd. and HITACHI Cable Ltd. in developing and producing the superconductors for the helical coils of LHD.

\section{REFERENCES}

[1] O. Motojima, K. Akaishi, K. Fujii, et al., "Physics and engineering design studies on the Large Helical Device," Fusion Eng. Des., vol. 20, 1993, pp. 3-14.

[2] T. Mito, K. Takahata, N. Yanagi, et al., "Short sample tests of aluminum-stabilized superconductors for Large Helical Device," Fusion Eng. Des., vol. 20, 1993, pp. 233-242.

[3] N. Yanagi, T. Mito, K. Takahata, et al., "Experimental observation of anomalous magnetoresistivity in $10-20 \mathrm{kA}$ class aluminum-stabilized superconductors for the Large Helical Device," Advances in Cryogenic Engineering-Materials, vol.40, 1994, pp. 459-468.

[4] N. Yanagi, T. Mito, S. Imagawa, et al., "Development, fabrication, testing and joints of aluminum stabilized superconductors for the helical coils of LHD," Proc. ICEC16/ICMC (KitaKyushu, 1996), Part 2, Elsevier, Oxford, 1997, pp. 751-754.

[5] P. W. Eckels, N.C. Iyer, A. Patterson, et al., "Magnetoresistance: the Hall effect in composite aluminum cryoconductors," Cryogenics, vol. 29, July 1989, pp. 748-752.

[6] H. Kaneko and N. Yanagi, "Enhancement of magnetoresistance due to Hall current in aluminum-copper composite," Cryogenics, vol.32, December 1992, pp. 1114-1120.

[7] J.M. Pfotenhauer, M.K. Abdelsalam, F. Bodker, et al., "Test results from SMES proof of principle experiment," IEEE Trans. Magn., vol. 27, 1991, pp. 1704-1707.

[8] X. Huang and Y.M. Eyssa, "Stability of large composite superconductors," IEEE Trans. Magn., vol. 27, 1991, pp. 23042307.

[9] S. Imagawa, N. Yanagi, T. Mito, et al., "Evaluation of recovery current of the helical coil for LHD," Proc. ICEC16/ICMC (Kita-Kyushu, 1996), Part 3, Elsevier, Oxford, 1997, pp. 18251828.

[10] K. Seo, M. Morita, and J. Yamamoto, "Stability of synthetic enameled superconducting wires," Proc. ICEC16/ICM C (Kita-Kyushu, 1996), Part 2, Elsevier, Oxford, 1997, pp. 12891292.

[11] S. Noguchi, A. Ishiyama, T. Satow, N. Yanagi, "Transient stability of large aluminum stabilized superconductors," this conference. 